\title{
Axiomatic Extensions of Höhle's Monoidal Logic
}

\author{
Esko Turunen \\ Tampere University of Technology
}

Tampere, Finland

\begin{abstract}
We introduce an axiomatic extension of Höhle's Monoidal Logic called Semi-divisible Monoidal Logic, and prove that it is complete by showing that semi-divisibility is preserved in MacNeille completion. Moreover, we introduce Strong semidivisible Monoidal Logic and conjecture that a predicate formula $\alpha$ is derivable in Strong Semi-divisible Monadic logic if, and only if its double negation $\neg \neg \alpha$ is derivable in Łukasiewicz* logic.
\end{abstract}

Keywords: Residuated lattice, non-classical logics, substructural logics.

\section{Introduction}

The origin of this paper is in our 2001 study (cf. [22]) on Hájek's BL-algebras (cf. [9]) where we observed that the set of complement elements, also called regular elements, of a BL-algebra $L$ is the largest sub MV-algebra of $L$. Thus, in this sense BL-algebras and MV-algebras are in the same relation that Heyting algebras and Boolean algebras are (cf. [8]) or, as proved in [22], SBL-algebras and Boolean algebras are. We introduced a subset $\left\{x^{*} \mid x \in L\right\}\left(=\left\{x^{* *} \mid x \in L\right\}\right)$, called an $M V$-center of $L$, and denoted it by $M V(L)$. In [22], [19], [20], [21] we used $M V(L)$ in analyzing BL-algebras and showed that many properties hold in a BL-algebra $L$ if, and only if they hold in the MV-center $M V(L)$.

Subsequently we concentrated our attention on generalized probability theory, see e.g. [4], [5], [6]. We got an impression that such generalization reduce extensively in probability theory on MValgebras introduced by Chovanec and Mundici (see [3] and [15], respectively) and published two papers where we showed that this indeed is the case (cf. $[14,23])$. More precisely, if the regular elements of a residuated lattice $L$ fulfill a certain extra condition, then the MV-center becomes an MV-algebra and states (i.e. additive measures of truth) and (maximal) filters on the $L$ are uniquely determined by states and (maximal) filters on $M V(L)$. However, it should be noticed that in general residuated lattice framework, $M V(L)$ is not an MV-subalgebra of $L$ but, using quantale theoretical terminology of [16], is a quotient w.r.t. the quantic nucleus given by the double negation.

In this paper we deal with non-classical logic.
When Ulrich Höhle introduced Monoidal predicate logic in $[10,11]$, his purpose was to outline a common framework for a diversity of monoidal structures which constitute the basis of various nonclassical logics. Indeed, Monoidal predicate logic connects Intuitionistic logic, Łukasiewicz* $\operatorname{logic}^{1}$ and (commutative) Linear logic or, putting it in other words, these logics are axiomatic extensions of Monoidal predicate logic. The main tool to prove the completeness of Monoidal logic and its various axiomatic extensions is to construct canonical model and show that if a formula is not provable, then it is not valid in this model. The canonical model is obtained by constructing first the corresponding Lindenbaum algebra and then its MacNeille completion.

We posed a question 'What is the most general axiomatic extension of Monoidal logic whose negative part is Eukasiewicz* logic'. Cignoli and Torrens $[1,2]$, see also [7], gave the following answers in sentential logic framework:

The following theorem, due to Glivenko, see also [13], shows that classical propositional logic can be interpreted in intuitionistic propositional logic.

Theorem [8] A formula $\varphi$ is provable in classical propositional logic iff the formula $\neg \neg \varphi$ is provable in intuitionistic propositional logic.

Nevertheless, intuitionistic logic is not the only substructural logic into which classical logic can be interpreted by double negation translation; there are other pairs of substructural logics that are related in the same way. Denote by SBL the extension of Hájek basic logic $\mathbf{B L}$ by the axiom $(\chi \cdot(\chi \rightarrow \neg \chi)) \rightarrow$ $\psi$.

Theorem [1, 2] (1) A formula $\varphi$ is provable in classical propositional logic iff the formula $\neg \neg \varphi$ is provable in SBL.

(2) A formula $\varphi$ is provable in Eukasiewicz infinitevalued propositional logic iff the formula $\neg \neg \varphi$ is provable in Hájek basic logic.

(3) Let $\mathbf{L}$ be an extension of $\mathbf{F L}_{\mathbf{e w}}$ that contains the axiom $\neg \neg(\neg \neg \psi \rightarrow \psi)$ and let $\mathbf{I n}(\mathbf{L})$ be extension of $\mathbf{L}$ by the axiom $\neg \neg \psi \rightarrow \psi$. Then a formula $\varphi$ is provable in $\mathbf{I n}(\mathbf{L})$ iff the formula $\neg \neg \varphi$ is provable

\footnotetext{
${ }^{1}$ Łukasiewicz infinite valued propositional logic is a complete logic while the Łukasiewicz predicate logic is not. Scarpellini [17] introduced in 1962 Łukasiewicz* logic by adding a new infinitary rule of inference and proved that this logic is a complete predicate logic.
} 
in $\mathbf{L}$.

The logic $\mathbf{F} \mathbf{L}_{\text {ew }}$ is equivalent to the propositional version of Höhle's Monoidal logic and the extension of $\mathbf{F L}_{\mathbf{e w}}$ that contains the axiom $\neg \neg(\neg \neg \psi \rightarrow \psi)$ would be strong Monoidal propositional logic in our terminology. Our intention is to define the most common substructural predicate logic L, i.e. an axiomatic extension of Monoidal predicate logic, such that a formula $\varphi$ is provable in $\mathbf{I n}(\mathbf{L})$ iff the formula $\neg \neg \varphi$ is provable in $\mathbf{L}$ where, in addition, $\operatorname{In}(\mathbf{L})$ is Łukasiewicz* predicate logic. However, this ultimate goal remains an open problem.

\section{Mathematical preliminaries}

A lattice $L=\langle L, \leq, \wedge, \vee, \mathbf{0}, \mathbf{1}\rangle$ with the least element $\mathbf{0}$ and the largest element $\mathbf{1}$ is called residuated if it is endowed with a couple of binary operations $\langle\odot, \rightarrow\rangle$ (called adjoint couple) such that $\odot$ is associative, commutative, isotone, $x \odot \mathbf{1}=x$ holds for all elements $x \in L$, and a residuation

$$
x \odot y \leq z \quad \text { iff } \quad x \leq y \rightarrow z
$$

holds for all elements $x, y, z \in L$. In [11], residuated lattices are called commutative, integral, residuated $\ell-$ monoids. Complete residuated lattices are examples of quantales (see [16]) i.e. complete lattices with an associative, supremum preserving operation $\odot$. We take here mainly a residuated lattice theoretical approach and refer to quantales only incidentally. In what follows we use the following equations known to hold in all residuated lattices (cf. [11], [18])

$$
x \rightarrow(y \rightarrow z)=(x \odot y) \rightarrow z=y \rightarrow(x \rightarrow z),
$$

in particular, for $z=\mathbf{0}$ and by setting $x^{*}=x \rightarrow \mathbf{0}$ we get

$$
\begin{array}{r}
x \rightarrow y^{*}=(x \odot y)^{*}=y \rightarrow x^{*}, \\
x \odot x^{*}=\mathbf{0}, \mathbf{0}^{*}=\mathbf{1}, \mathbf{1}^{*}=\mathbf{0}, \\
\text { if } x \leq y \text { then } y^{*} \leq x^{*}, \\
x^{*} \leq y^{*} \text { iff } y^{* *} \leq x^{* *}, \\
x \leq x^{* *}, \\
x^{*}=x^{* *}, \\
x^{*} \rightarrow y^{*}=y^{* *} \rightarrow x^{* *} .
\end{array}
$$

Since the operation $\odot$ preserves joins in $L,\left(x \vee y^{*}\right) \odot$ $y=(x \odot y) \vee\left(y^{*} \odot y\right)=x \odot y$ and, therefore

$$
x \vee y^{*} \leq y \rightarrow(x \odot y)
$$

Moreover, in any residuated lattice $L$ holds

$$
\left(\bigvee_{i \in \Gamma} x_{i}\right)^{*}=\bigwedge_{i \in \Gamma} x_{i}^{*}
$$

whenever these meets and joins exist in $L$. A residuated lattice $L$ is

- a (commutative) Girard monoid if the complement is involutive, i.e., $x=x^{* *}$ for all $x \in L$. Complete Girard monoids - that is, Girard monoids whose lattice structure contains all meets and joins - are called Girard quantales.

- a Heyting algebra if the product operation $\odot$ coincides with the operation $\wedge$.

- called divisible if, for all $x, y \in L, x \leq y$, there is $z \in L$ such that $x=z \odot y$.

- called prelinear if $(x \rightarrow y) \vee(y \rightarrow x)=\mathbf{1}$ holds for all $x, y \in L$; prelinearity is also called Strong de Morgan Law in [11]. Prelinearity is equivalent to a condition $(x \wedge y) \rightarrow z=(x \rightarrow z) \vee(y \rightarrow z)$ for all $x, y, z \in L$. In particular, in prelinear residuated lattices $(x \wedge y)^{*}=x^{*} \vee y^{*}$ for all $x, y \in L$. Prelinear residuated lattices are also called $M T L$-algebras.

- a $B L$-algebra if it is prelinear and divisible. $S B L$ algebras are BL-algebras with an additional property $(x \odot y)^{*}=x^{*} \vee y^{*}$ for all $x, y \in L$ - there are several equivalent definitions in literature.

- an $M V$-algebra if it is a divisible Girard monoid or, equivalently, a $B L$-algebra with an involutive complement or, equivalently, if $x \vee y=(y \rightarrow x) \rightarrow x$ holds for any $x, y \in L$.

- called IMTL-algebra if it is an involutive MTLalgebra.

In this paper we are interested in complete semidivisible residuated lattices ${ }^{2} L$ (cf. [23]), that is, for all $x, y \in L$,

$$
\left(x^{*} \rightarrow y^{*}\right) \rightarrow y^{*}=\left(y^{*} \rightarrow x^{*}\right) \rightarrow x^{*} .
$$

It was proved in [23] that condition (11) is equivalent to the condition that, for all $x, y \in L$ holds

$$
\left(x^{*} \wedge y^{*}\right)^{*}=\left[x^{*} \odot\left(x^{*} \rightarrow y^{*}\right)\right]^{*} .
$$

In particular, if for all $x, y \in L$ holds

$$
x \wedge y=x \odot(x \rightarrow y)
$$

then $L$ is divisible. Heyting algebras and BLalgebras are divisible. We also study semi-divisible residuated lattices $L$ which are strong, that is, for all $x, y \in L$,

$$
\left(x^{* *} \rightarrow x\right)^{* *}=\mathbf{1} .
$$

Assuming that (11) holds, a subset $M V(L)=$ $\left\{x^{*} \mid x \in L\right\}$ (non-void as $\mathbf{0 , 1} \in M V(L)$ and called the $M V$-center of $L)$ induces an $M V$-algebra, where the operations $\odot_{M V}$ and $\vee_{M V}$ are defined via

$$
\begin{aligned}
x^{*} \odot_{M V} y^{*} & =\left(x^{*} \odot y^{*}\right)^{* *}, \\
x^{*} \vee_{M V} y^{*} & =\left(x^{*} \vee y^{*}\right)^{* *} .
\end{aligned}
$$

The order $\leq$ and the operation $\rightarrow$ is that of $L$; e.g. for the residuum $\rightarrow$ we can see this as follows. First, for any $x, y, z \in L, x^{*} \rightarrow y^{*}=\left(x^{*} \odot y\right)^{*} \in M V(L)$ and second, $x^{*} \leq y^{*} \rightarrow z^{*}$ iff $x^{*} \odot y^{*} \leq z^{*}$ iff

\footnotetext{
${ }^{2}$ The anonymous reviewer remarks that the name semidivisible in this context might be confusing. However, we have not invented anything better.
} 
$\left(x^{*} \odot y^{*}\right)^{* *} \leq z^{*(* *)}$ iff $x^{*} \odot \odot_{M V} y^{*} \leq z^{*}$. Since the residuum w.r.t $\odot_{M V}$ is unique the assertion holds. Moreover, the operations ${ }^{*}, \wedge$ in $M V(L)$ are those of $L$.

Notice that if $L$ is a semi-divisible residuated lattice then $M V(L)$ is divisible. Indeed, for any $x^{*}, y^{*} \in M V(L)$,

$$
\begin{gathered}
x^{*} \wedge y^{*}=\left(x^{*} \wedge y^{*}\right)^{* *}=\left[x^{*} \odot\left(x^{*} \rightarrow y^{*}\right)\right]^{* *}= \\
x^{*} \odot_{M V}\left(x^{*} \rightarrow y^{*}\right) .
\end{gathered}
$$

Moreover, $x^{*} \vee_{M V} y^{*}=\left(y^{*} \rightarrow x^{*}\right) \rightarrow x^{*}$. In fact, the $M V$-center (the set of regular elements) can be seen as a particular case of a more general concept (cf. [16]), namely

Definition Assume $L$ is a residuated lattice. A mapping $\mu: L \mapsto L$ is a nucleus of $L$ if, for all $x, y \in L$,

(i) $\mu$ is isotone: if $x \leq y$ then $\mu(x) \leq \mu(y)$,

(ii) $x \leq \mu(x)$,

(iii) $\mu$ is idempotent: $\mu(x)=\mu(\mu(x))$,

(iv) $\mu(x) \odot \mu(y) \leq \mu(x \odot y)$.

If $\mu$ is a nucleus, then the range of $\mu$ is denoted by

$$
L_{\mu}=\{\mu(x) \mid \quad x \in L\} \quad(\subseteq L) .
$$

A residuated structure on $L_{\mu}$ is introduced in the following way:

$1^{\circ}$ The partial order $\leq$ is that of $L$. Then $\sup \{\mu(x), \mu(y)\}, \inf \{\mu(x), \mu(y)\} \in L_{\mu}$ are given by $\mu(x) \sqcup \mu(y)=\mu(\mu(x) \vee \mu(y))$ and

By simple reasoning one verifies that general joins and meets in $L_{\mu}$ are given by

$\sqcup_{i \in \Gamma} \mu\left(a_{i}\right)=\mu\left(\bigvee_{i \in \Gamma} \mu\left(a_{i}\right)\right), \sqcap_{i \in \Gamma} \mu\left(a_{i}\right)=\bigwedge_{i \in \Gamma} \mu\left(a_{i}\right)$

whenever the corresponding joins and meets exist in $L$.

$2^{\circ}$ A product operation $\odot_{\mu}$ on $L_{\mu}$ is defined via

$$
\mu(x) \odot_{\mu} \mu(y)=\mu(\mu(x) \odot \mu(y)) .
$$

Moreover, following [16] it is an easy exercise to show that the following proposition holds (an alternative reference is [7]).

Proposition The operation $\odot_{\mu}$ defined by (18) is commutative and $\mathbf{0}_{\mu}=\mu(\mathbf{0}), \mathbf{1}_{\mu}=\mu(\mathbf{1})=\mathbf{1}$ are the least and largest elements of $L_{\mu}$, respectively. Moreover, the operation $\odot_{\mu}$ is associative and isotone and there is a residuation $\mu(x) \odot_{\mu} \mu(y) \leq \mu(z)$ iff $\mu(x) \leq \mu(y) \rightarrow \mu(z)$.

Thus, given a residuated lattice $L=\langle L, \leq$ $, \wedge, \vee, \odot, \rightarrow, \mathbf{0}, \mathbf{1}\rangle$ and a nucleus $\mu$ on $L$, the structure $L_{\mu}=\left\langle L_{\mu}, \leq, \sqcup, \sqcap, \odot_{\mu}, \rightarrow, \mathbf{0}_{\mu}, \mathbf{1}\right\rangle$ as defined above is a residuated lattice, called the quotient of $L$ w.r.t. $\mu$. Moreover, the nucleus $\mu: L \mapsto L_{\mu}$ is a surjective monoid-homomorphism.

Next consider a mapping $\mu_{\neg \neg}: L \mapsto L$ such that $\mu_{\neg \neg}(x)=x^{* *}$. It is obvious that such a mapping fulfills conditions (i) - (iii) of 2 . It has the property (iv), too. Indeed, $(x \odot y)^{*}=y \rightarrow(x \rightarrow \mathbf{0})=y \rightarrow$ $\left(x^{* *} \rightarrow \mathbf{0}\right)=x^{* *} \rightarrow y^{*}$. Thus $(x \odot y)^{*} \odot x^{* *} \leq y^{*}$. Therefore $y^{* *} \leq\left[(x \odot y)^{*} \odot x^{* *}\right]^{*}=x^{* *} \rightarrow(x \odot y)^{* *}$, hence $y^{* *} \odot x^{* *} \leq(x \odot y)^{* *}$, which is condition (iv).

Since $x^{* * *}=x^{*}$ for all $x^{*} \in M V(L)$ and as the $M V$-center $M V(L)$ of a residuated lattice $L$ can be given also by $M V(L)=\left\{x^{* *} \mid x \in L\right\}$, we conclude

Theorem For any residuated lattice $L$, the set $M V(L)$ equipped with the above defined operations is the quotient $\mu_{\neg \neg}(L)$ of $L$ w.r.t. the nucleus determined by double negation. Moreover, $M V(L)$ is a (commutative) Girard monoid.

It is proved in [12] that in a Heyting algebra $L$, the nucleus determined by double negation is a subalgebra of $L$ and is a Boolean algebra iff $(x \wedge y)^{*}=x^{*} \vee y^{*}$ for all $x, y \in L$ iff $x^{*} \vee x^{* *}=\mathbf{1}$ for all $x \in L$.

We proved in [22] that in $B L$-algebras the quotient $M V(L)$ is a subalgebra of $L$; this was established independently also in [1]. However, our proof of this fact rests upon the representation theorem of $B L$-algebras (cf. [9]). We have a new direct proof to

Proposition Assume $L$ is a BL-algebra. Then $a^{* *} \odot b^{* *}=a^{* *} \odot_{M V} b^{* *}$ for any $a, b \in L$ i.e. $M V(L)$ is a subalgebra of $L$.

In Girard monoids and, in particular in IMTLalgebras, the operations $\odot$ and $\odot_{M V}$ trivially coincide, and $M V(L)$ is a subalgebra of $L$. However, it is an open probem to give necessary and sufficient conditions such that the operation $\odot_{M}$ coincides with $\odot$.

We may characterize semi-divisible residuated lattices by the following

Proposition $A$ residuated lattice $L$ is semidivisible if, and only if the quotient $M V(L)$ w.r.t. the double negation is an $M V$-algebra.

Involutive residuated lattices are trivially strong. We have

Proposition Any divisible residuated lattice is strong.

In particular, Heyting algebras and BL-algebras are strong. Semi-divisible residuated lattices are not necessary strong; in [23] we introduced a semidivisible residuated lattice $L_{s D}$ on the unit real interval $[0,1]$ such that

$$
\begin{gathered}
x \odot y= \begin{cases}0 & \text { if } x, y \in\left[0, \frac{1}{2}\right], \\
\min \{x, y\} & \text { otherwise. }\end{cases} \\
x \rightarrow y= \begin{cases}1 & \text { if } x \leq y, \\
\frac{1}{2} & \text { if } y<x \leq \frac{1}{2}, \\
y & \text { if } y<x, \frac{1}{2} \leq x .\end{cases}
\end{gathered}
$$

$L_{s D}$ is not strong: for example $\left[\left(\frac{1}{3}\right)^{* *} \rightarrow \frac{1}{3}\right]^{* *}=\frac{1}{2}$.

Cignoli and Torrens [1] proved that the nucleus $\mu_{\neg \neg}: L \mapsto L$ gives a homomorphism from $L$ onto $M V(L)$, that is 


$$
(x \rightarrow y)^{* *}=x^{* *} \rightarrow y^{* *}
$$

if, and only if $L$ is strong. Notice that in a residuated lattice, for all $x, y, z \in L, x \leq y \rightarrow(x \odot y)$, therefore $x^{* *} \leq[y \rightarrow(x \odot y)]^{* *}$, hence $L$ being strong, $x^{* *} \leq y^{* *} \rightarrow(x \odot y)^{* *}$, whence $x^{* *} \odot y^{* *} \leq$ $(x \odot y)^{* *}$. Thus $x^{* *} \odot_{M V} y^{* *} \leq(x \odot y)^{* *}$. Since the converse trivially holds we have

$$
(x \odot y)^{* *}=x^{* *} \odot_{M V} y^{* *} .
$$

We conclude that, in strong residuated lattices, the nucleus $\mu_{\neg \neg}: L \mapsto M V(L)$ is a homomorphism w.r.t. the product operation, too.

\section{MacNeille completion of a residuated lattice}

Recall from [11] some definitions and basic properties of MacNeille completion $L^{\sharp}$ of a residuated lattice $L$, which will play a crucial role when proving the completeness of Monoidal logic and its axiomatic extensions. Assume $\langle L, \leq\rangle$ is a partially ordered set and $A \subseteq L$. Denote

$1^{\circ} \mathcal{U}(A)=\{x \in L \mid a \leq x$ for all $a \in A\}$, the set of all upper bounds of $A$,

$2^{\circ} \mathcal{L}(A)=\{y \in L \mid \quad y \leq a$ for all $a \in A\}$, the set of all lower bounds of $A$,

$3^{\circ} A^{\sharp}=\mathcal{L}(\mathcal{U}(A))$ (read sharp $\left.A\right)$.

Denote $L^{\sharp}=\left\{A^{\sharp} \quad A \subseteq L\right\}$. Define a mapping $j$ : $L \mapsto L^{\sharp}$ via $j(a)=\{a\}^{\sharp}$. Then $j$ is an embedding and we have

Proposition [Lemma 5.4 in [11]] $\left\langle L^{\sharp}, \subseteq\right\rangle$, the MacNeille completion of a poset $\langle L, \leq\rangle$, is a complete lattice.

Theorem[Uniqueness of MacNeille completion, Theorem 5.5 in [11]] Let $\langle P, \leq\rangle$ be a partially ordered set. Then there is a further partially ordered set $\langle L, \leq\rangle$ and a mapping $j: P \mapsto L$ satisfying the following conditions:

$1^{\circ} j$ is an embedding - i.e. $j(a) \leq j(b)$ iff $a \leq b$, $2^{\circ}\langle L, \leq\rangle$ is a complete lattice,

$3^{\circ}$ For every element $\ell \in L$, the following holds

(a) $\{a \in P \mid j(a) \leq \ell\}^{\sharp}=\{a \in P \mid j(a) \leq \ell\}$,

(b) $\ell=\bigvee\{j(a) \mid a \in P, j(a) \leq \ell\}$.

Moreover, $\langle j,\langle L, \leq\rangle\rangle$ is uniquely determined by $1^{\circ}$ $3^{\circ}$ up to an order isomorphism.

Theorem[cf. page 85 in [11]] For any residuated lattice L, its MacNeille completion $L^{\sharp}$ is a complete residuated lattice, where

$$
A^{\sharp} \odot \odot^{\sharp} B^{\sharp}=\{a \odot b \mid \quad a \in A, b \in B\}^{\sharp} .
$$

and the residuum is given via $A^{\sharp} \rightarrow \sharp \quad B^{\sharp}=$ $\bigvee\left\{C^{\sharp} \mid C^{\sharp} \odot \odot^{\sharp} A^{\sharp} \subseteq B^{\sharp}\right\}$.

The residuated structure is preserved in the formation of MacNeille completion:

Proposition [Theorem 5.7 in [11]] The embed$\operatorname{ding} j: L \mapsto L^{\sharp}$ is a homomorphism, i.e., $j(a \odot b)=$ $j(a) \odot^{\sharp} j(b)$ and $j(a \rightarrow b)=j(a) \rightarrow^{\sharp} j(b)$.
Theorem [Corollary 5.9, Theorem 5.12 and Theorem 6.3 in [11], respectively] (i) MacNeille completion preserves the double negation law. (ii) Assume $L$ is a divisible residuated lattice such that, for all $x, y \in L$, the following holds

$$
\text { if } x \leq y^{n} \text { for all natural } n \text {, then } x=x \odot y \text {. }
$$

Then $L^{\sharp}$ is divisible. (iii) The MacNeille completion of an $M V$-algebra $L$ is an $M V$-algebra iff $L$ is semi-simple. (iv) Semi-simplicity of an $M V$ algebra implies condition (19).

We have proved the following two new results on MacNeille completion of semi-divisible residuated lattices.

Theorem Let $L$ be a residuated lattice and $L^{\sharp}$ its MacNeille completion. Then the MacNeille completion of $M V(L)$ is $M V\left(L^{\sharp}\right)$ and the embedding $i$ : $M V(L) \mapsto M V\left(L^{\sharp}\right)$ is the restriction of $j: L \mapsto L^{\sharp}$.

Theorem Assume $L$ is a semi-divisible residuated lattice. Then the following are equivalent conditions

(a) The MacNeille completion $L^{\sharp}$ is a semi-divisible residuated lattice i.e. $M V\left(L^{\sharp}\right)$ is an $M V$-algebra.

(b) For all $x, y \in L$, if $x^{*} \leq\left(y^{*}\right)^{n}$ for all natural $n$ then $x^{*}=x^{*} \odot_{M V} y^{*}$, where the power $n$ is taken w.r.t. the operation $x^{*} \odot_{M V} y^{*}=\left(x^{*} \odot y^{*}\right)^{* *}$.

(c) $M V(L)$ is a semi-simple $M V$-algebra.

\section{Monoidal logic and its axiomatic extensions}

Definition $[10,11]$ The formalized predicate language $\mathcal{L}$ of Monoidal logic consists of (i) $n$-ary predicates $P_{1}, \cdots, P_{m}$, (ii) an infinite sequence $x_{1}, \cdots, x_{m}, \cdots$ of individual variables, (iii) logical connectives $\neg$ (negation), $\underline{\wedge}$ (conjunction), $\underline{\vee}$ (disjunction), \& (bold conjunction) and $\Rightarrow$ (implication), where $\neg$ is a unary connective and the remaining ones are binary, (iv) quantifiers $\forall$ (for all) and $\exists$ (exists). Atomic formulae are the symbols $P\left(x_{1}, \cdots x_{n}\right)$, where $P$ is an $n$-ary predicate and $x_{1}, \cdots x_{n}$ are variables. Atomic formulae are in the set $\mathcal{F}$ of well formed formulae and if $\alpha, \beta \in \mathcal{F}$, then $\neg \alpha, \alpha \underline{\wedge}, \alpha \underline{\vee} \beta, \alpha \& \beta, \alpha \Rightarrow \beta, \forall x \alpha, \exists x \alpha \in \mathcal{F}$. Free and bound variables are defined as in Classical Predicate logic.

Let $L$ be a complete residuated lattice. The $L$ valued interpretations of Monoidal logic are introduced in [10] in the following way. Assume $X$ is a non-void set called the universe. Let $V$ be the set of all individual variables of the language $\mathcal{L}$. A map $v: V \mapsto X$ is called valuation. Moreover, consider a mapping $\Phi$ such that $\Phi$ assigns to every $n$-ary predicate symbol $P$ a map $\Phi(P): X^{n} \mapsto L$. Then, for any valuation $v$, the $L$-valued interpretations are mappings $\Theta_{v}: \mathcal{F} \mapsto L$ first defined for atomic formulas via

$$
\Theta_{v}\left(P\left(x_{1}, \cdots, x_{n}\right)\right)=\Phi(P)\left(v\left(x_{1}\right), \cdots, v\left(x_{n}\right)\right),
$$


and then extended recursively into the whole $\mathcal{F}$ as follows

$$
\begin{gathered}
\Theta_{v}(\neg \alpha)=\Theta_{v}(\alpha) \rightarrow \mathbf{0}, \\
\Theta_{v}(\alpha \Rightarrow \beta)=\Theta_{v}(\alpha) \rightarrow \Theta_{v}(\beta), \\
\Theta_{v}(\alpha \& \beta)=\Theta_{v}(\alpha) \odot \Theta_{v}(\beta), \\
\Theta_{v}(\alpha \underline{\wedge} \beta)=\Theta_{v}(\alpha) \wedge \Theta_{v}(\beta), \\
\Theta_{v}(\alpha \underline{\vee} \beta)=\Theta_{v}(\alpha) \vee \Theta_{v}(\beta), \\
\Theta_{v}((\forall x) \alpha)=\bigwedge_{x \in X} \Theta_{v_{x}}(\alpha), \\
\Theta_{v}((\exists x) \alpha)=\bigvee_{x \in X} \Theta_{v_{x}}(\alpha), \\
\text { where } v_{x}(y)=\left\{\begin{array}{cc}
v(y) & \text { if } x \neq y \\
x & \text { if } x=y .
\end{array}\right.
\end{gathered}
$$

A well formed formula $\alpha$ is called $L$-valid if $\Theta_{v}(\alpha)=$ 1 for any valuation $v$ and any mapping $\Phi$. If $\alpha$ is $L$-valid for any complete residuated lattice $L$, then $\alpha$ is called valid; this situation is denoted by writing $=\alpha$. The following Theorem is obvious

Theorem In Monoidal logic holds, for any well formed formula $\alpha, \beta, \gamma$ :

$$
\begin{array}{r}
\models(\alpha \Rightarrow \beta) \Rightarrow[(\beta \Rightarrow \gamma) \Rightarrow(\alpha \Rightarrow \gamma)] \\
\models \alpha \Rightarrow(\alpha \underline{\vee} \beta) \\
\models \beta \Rightarrow(\alpha \underline{\vee} \beta) \\
\models((\alpha \underline{\vee}) \Rightarrow \gamma))] \\
\models(\alpha \wedge \beta) \Rightarrow \alpha \\
\models(\alpha \& \beta) \Rightarrow \alpha \\
\models(\alpha \underline{\wedge} \Rightarrow \beta \\
\models(\alpha \& \beta) \Rightarrow(\beta \& \alpha) \\
\models(\alpha \Rightarrow \gamma) \Rightarrow(\alpha \&(\beta \& \gamma)) \\
\models(\gamma \Rightarrow \alpha) \Rightarrow[(\gamma \Rightarrow \beta) \Rightarrow(\gamma \Rightarrow(\alpha \wedge \beta))] \\
\models((\alpha \Rightarrow(\beta \Rightarrow \gamma)) \Rightarrow[(\alpha \& \beta) \Rightarrow \gamma] \\
\models((\alpha \& \beta) \Rightarrow \gamma) \Rightarrow[(\alpha \Rightarrow(\beta \Rightarrow \gamma)] \\
\models(\alpha \& \neg \alpha) \Rightarrow \beta \\
\models(\alpha \Rightarrow(\alpha \& \neg \alpha)) \Rightarrow \neg \alpha \\
\models(\forall x) \alpha \Rightarrow \alpha(x / y) \\
\models \alpha(x / y) \Rightarrow(\exists x) \alpha
\end{array}
$$

where $y$ is an individual variable for which $x$ is free in $\alpha$.

If we add some extra conditions for the residuated lattices $L$ in the definition of $L$-valued interpretations, we obtain special cases of Monoidal logic. Originally in $[10,11]$ the following axiomatic extension of Monoidal logic was introduced. Assume $L$ is any complete $M V$-algebra. Then the extension is called Eukasiewicz* logic and all the valid formulae are called $M V$-valid. In Łukasiewicz* logic holds

$$
\begin{array}{r}
\models \neg \neg \alpha \Rightarrow \alpha \\
\models(\alpha \underline{\wedge}) \Rightarrow[\alpha \&(\alpha \Rightarrow \beta)]
\end{array}
$$

We introduce the following axiomatic extensions of Monoidal predicate logic. Assume $L$ is any complete semi-divisible residuated lattice. Then the extension is called Semi-divisible Monoidal logic and all the valid formulae are called semi-divisible valid. In Semi-divisible Monoidal logic holds, for any well formed formulae $\alpha, \beta$,

$\models[(\neg \alpha \Rightarrow \neg \beta) \Rightarrow \neg \beta)] \Rightarrow[(\neg \beta \Rightarrow \neg \alpha) \Rightarrow \neg \alpha)]$

Moreover, assume $L$ is any complete strong semidivisible residuated lattice. Then the extension is called Strong semi-divisible Monoidal logic and all the valid formulae are called strong semi-divisible valid. In Strong semi-divisible Monoidal logic holds, for any well formed formulae $\alpha$,

$$
\models \neg \neg(\neg \neg \alpha \Rightarrow \alpha)
$$

Logical axioms of Monoidal logic are the schemas (20) - (35). In particular, if we focus on Semidivisible Monoidal logic or Strong semi-visible Monoidal logic, we assume the corresponding axiom schemas (38) or (38) and (39), too. Rules of inference of Monoidal logic are Modus Ponens and the usual Quantifier rules

$(\forall)$ From $(\alpha \Rightarrow \beta)$ infer $(\alpha \Rightarrow(\forall x) \beta)$ provided $x$ is not free in $\alpha$,

( $\exists$ ) From $(\alpha \Rightarrow \beta)$ infer $((\exists x) \alpha \Rightarrow \beta)$ provided $x$ is not free in $\beta$.

A well formed formula $\alpha$ is provable if $\alpha$ is of a form of axiom schema or $\alpha$ results from finite many applications of rules of inference to provable well formed formulae. If $\alpha$ is provable, then this situation is denoted by $\vdash \alpha$. Monoidal logic as well as its axiomatic extensions introduced by Höhle are sound:

for all well formed formulae $\alpha, \vdash \alpha$ implies $\models \alpha$.

This follows by the simple fact that all axiom schemas are valid and rules of inference preserve validity. Completeness, i.e. that

for all well formed formulae $\alpha, \models \alpha$ implies $\vdash \alpha$

is obtained by means of a canonical model: first construct the corresponding Lindenbaum algebra $L$ and prove that $L$ is a residuated lattice (or a suitable special residuated lattice) and then consider its MacNeille completion $L^{\sharp}$; if $\forall \alpha$, then $\alpha$ is not $L^{\sharp}$ valid. Recalling that the double negation law and idempotency of product are preserved in the MacNeille completion, the following holds

Theorem [Theorem 4.1 in [10]] Monoidal logic is complete w.r.t. complete residuated lattices. (Commutative) Linear logic is complete w.r.t. Girard quantales. Intuitionistic logic is complete w.r.t. complete Heyting algebras.

For axiomatic extensions of Monoidal logic containing divisibility or semi-divisibility axioms, equivalents to the previous Theorem do not hold as such. By [10], a necessary and sufficient condition to obtain completeness of Łukasiewicz* logic is that the corresponding Lindenbaum algebra is a semisimple $M V$-algebra. This can be done by adding a new infinitary inference rule

$$
\text { From }[\neg \alpha \Rightarrow \alpha \& \cdots \& \alpha] \forall n \text { infer } \alpha \text {. }
$$


Then we have

Theorem [Theorem 4.2 in [10]] Eukasiewicz* logic is complete w.r.t. complete $M V$-algebras.

Similarly, to obtain completeness of Semidivisible Monoidal logic, it is necessary and sufficient that we add another infinitary inference rule

$$
\text { From }[\neg \neg \alpha \Rightarrow \neg \alpha \underline{\underline{L}} \cdots \underline{\&} \neg \alpha] \forall n \text { infer } \neg \alpha,
$$

where the new logical connective $\underline{\&}$ is defined recursively via $\neg \alpha \underline{\underline{\alpha}} \neg \beta=\neg \neg[\neg \alpha \& \neg \beta]$.

Then we have

Theorem Semi-divisible Monoidal logic is complete w.r.t. complete semi-divisible residuated lattices.

However, we have not been able to prove or disprove that strongness is preserved under MacNeille completion. If this conjecture is true, then we have

Theorem Strong semi-divisible Monoidal logic is complete w.r.t. complete strong semi-divisible residuated lattices.

Theorem A predicate formula $\alpha$ is derivable in Strong Semi-divisible Monadic logic if, and only if its double negation $\neg \neg \alpha$ is derivable in Eukasiewic $z^{*}$ logic.

\section{References}

[1] Cignoli, R. and Torrens A.: Hájek basic fuzzy logic and Łukasiewicz infinite-valued logic. Arch. Math. Logic 42(2003) 361-370.

[2] Cignoli, R. and Torrens A.: Glivenko like theorems in natural expansions of $B C K$-logic. Math. Log. Quart. 50(2004) 111-125.

[3] Chovanec, F.: States and observables on $M V$ algebras, Tatra Mt. Math. Publ. 3(1993), 467490.

[4] Dvurečenskij, A.: States on Pseudo-MValgebras. Studia Logica 68(2001), 301-327.

[5] Dvurečenskij, A. and Rachunek, J.: On Riečan and Bosbach States for Bounded Non-commutative $R \ell$-Monoids. Math. Slovaca 56(2006), 487-500.

[6] Dvurečenskij, A. and Rachunek, J.: Probabilistic averaging in bounded commutative residuated 1-monoids. Discrete Mathematics 306(2006), 1317-1326.

[7] Galatos, N., Jipsen, P., Kowalski, T. and Ono, H.: Residuated Lattices: An Algebraic Glimpse at Substructural Logics, Studies in Logic and the Foundations of Mathematics. Vol 151. Elsevier, Amsterdam (2007).

[8] Glivenko, V.: Sur quelques points de la logique de M. Brower. Bull. Acad. des Sci. de Belgique 15(1929), 183-188.

[9] Hájek, P.: Metamathematics of Fuzzy Logic, Trends in Logic, Studia Logica Library, Kluwer, Dordrecht, Volume 4 (1998).

[10] Höhle, U.: Monoidal logic, in: Kruse, R., Gebhardt, J., and Palm, R.(eds.): Fuzzy Systems in
Computer Science, Vieweg, Wiesbaden, (1994) 233-243.

[11] Höhle, U.: Commutative, residuated $\ell$ monoids. In Höhle, U. and Klement, E. (eds), Non-Classical Logics and Their Applications to Fuzzy Subsets. Kluwer, Dordrecht (1995) 53106.

[12] Johnstone, P. T.: Stone spaces. Cambridge university press (1982).

[13] Kolmogorov, A.: On the principle of excluded middle (in Russian), Matematicheski Spornik 32(1925), 646-667.

[14] Mertanen, J. and Turunen, E: States on semidivisible generalized residuated lattices reduce to states on MV-algebras. Fuzzy Sets and Systems 159(2008), 3051-3064.

[15] Mundici, D.: Averaging the Truth-Value in Łukasiewicz Logic, Studia Logica 55(1995), 113127.

[16] Rosenthal, K. : Quantales and their applications. Pitman Research Notes in Mathematics Series 234. New York (1990).

[17] Scarpellini, B.: Die Nichtaxiomatisierbarkeit des unendlichen Prädikaten kalkulüs von Łukasiewicz. Journal of Symbolic Logic 27(1962) 159-170.

[18] Turunen, E.: Mathematics Behind Fuzzy Logic, Advances in Soft Computing, PhysicaVerlag, Heidelberg (1999).

[19] Turunen, E.: Boolean deductive systems of BL-algebras. Int. Jour.of Multiple-valued Logic 6(2001) 229-249.

[20] Turunen, E.: Semilocal BL-algeras. Proceedings of the IX International IFSA Congress 2005. 28.-31. July 2005, Beijing, China, 252-256.

[21] Turunen, E.: Semi-Boolean and Hyper Archimedean BL-algebras. Proceedings of the X International IFSA Congress 2007. 18.-21. June, 2007. Cancun, Mexico.419-426.

[22] Turunen, E. and Sessa, S.: Local $B L$-algebras, Int. Jour.of Multiple-valued Logic 6(2001), 229 249.

[23] Turunen, E. and Mertanen, J.: States on semi-divisible residuated lattices. Soft Computing 12(2007) 353-357 . 